\title{
Angiotensin II receptor blockade inhibits acute glomerular injuries with the alteration of receptor expression
}

\author{
Akiko Mii ${ }^{1,2}$, Akira Shimizu ${ }^{1}$, Yukinari Masuda ${ }^{1}$, Masamichi Ishizaki ${ }^{1}$, Hiroshi Kawachi ${ }^{3}$, Yasuhiko lino $^{2}$, \\ Yasuo Katayama ${ }^{2}$ and Yuh Fukuda ${ }^{1}$
}

Angiotensin II receptor blockade (ARB) suppresses the progression of chronic kidney disease. However, the renoprotective effect of ARB in the active phase of glomerulonephritis (GN) has not been evaluated in detail. We examined the alteration of angiotensin II receptors' expression and the action of ARB on acute glomerular injuries in GN. Thy-1 GN was induced in rats that were divided into three groups $(n=7$, in each group); high dose $(3 \mathrm{mg} / \mathrm{kg} / \mathrm{day})$ or low dose $(0.3 \mathrm{mg} / \mathrm{kg} /$ day) olmesartan (Thy-1 GN + HD- or LD-ARB group), and vehicle (Thy-1 GN group). Renal function and histopathology were assessed by week 2 . In the Thy-1 GN group, diffuse mesangiolysis and focal aneurysmal ballooning developed by day 3. Marked mesangial proliferation and activation progressed with glomerular epithelial injury. We confirmed that both angiotensin II type 1 receptor (AT1R) and type 2 receptor (AT2R) were expressed on glomerular endothelial, mesangial, epithelial cells, and macrophages, and increased 7 days after disease induction. However, ARB treatment caused a decrease in AT1R and a further increase in AT2R expression in glomeruli. ARB prevented capillary destruction and preserved eNOS expression after diffuse mesangiolysis. Mesangial proliferation and activation was suppressed markedly with low levels of PDGF-B expression. Glomerular desmin expression, which is a marker for injured glomerular epithelial cells, was diminished significantly with retained expression of nephrin and podoplanin. Glomerular macrophage infiltration was also inhibited. Proteinuria was suppressed significantly. Furthermore, these effects of ARB showed dose dependency. These results provide insights that ARB affects individual glomerular cells and macrophages through angiotensin II receptors, with the alteration of both AT1R and AT2R expressions, and leads to inhibition of the acute destructive and proliferative glomerular lesions in GN.

Laboratory Investigation (2009) 89, 164-177; doi:10.1038/labinvest.2008.128; published online 12 January 2009

KEYWORDS: angiotensin II type 1 receptor; angigotensin II type 2 receptor; ARB; Thy-1 glomerulonephritis; acute glomerular injury; renoprotection

The renin-angiotensin system (RAS) is a major regulatory system of cardiovascular and renal function. Classically, the main role of the RAS is maintaining electrolyte balance, body fluid volume, and blood pressure (BP) through angiotensin II-induced vasoconstriction and aldosterone release. ${ }^{1}$ In addition to its systemic hemodynamic effects, angiotensin II has several pathophysiological actions mediated by angiotensin II type I receptor (AT1R) and type 2 receptor (AT2R) in cardiovascular, atherosclerotic, and renal diseases. ${ }^{2}$ In the kidney, activation of the angiotensin II-AT1R pathway results in glomerular hypertension, production of pro-inflammatory mediators, cell proliferation, extracellular matrix synthesis, and release of reactive oxygen species, that facilitate kidney damage and advance chronic kidney disease. ${ }^{3-7}$

RAS blockade exhibits renoprotective effects, including anti-inflammatory, anti-proliferative, and anti-oxidative stress responses. These effects are greater than expected from their ability to lower BP alone. Clinical studies and basic research have revealed that AT1R blockade (ARB), as well as angiotensin-converting enzyme (ACE) inhibitors, suppresses the progression of chronic kidney disease. ${ }^{4,8-10}$ ARB inhibits the development of glomerular sclerosis and fibrosis with the prevention of proteinuria and renal dysfunction in the chronic phase of renal diseases. However, no detailed analyses

${ }^{1}$ Department of Analytic Human Pathology, Nippon Medical School, Tokyo, Japan; ${ }^{2}$ Department of Internal Medicine (Division of Neurology, Nephrology and Rheumatology), Nippon Medical School, Tokyo, Japan and ${ }^{3}$ Cell Biology Institute of Nephrology, Niigata University Graduate School, Niigata, Japan Correspondence: Dr A Shimizu, MD, PhD, Department of Analytic Human Pathology, Nippon Medical School, 1-25-2, Nezu, Bunkyo-ku, Tokyo 113-0031, Japan. E-mail: ashimizu@nms.ac.jp 
of $A R B$ action on the active phase of glomerulonephritis (GN) were presented. In addition, the alteration of glomerular AT1R and AT2R expressions in proliferative GN and the effects of ARB on these receptors' expression have not been clarified.

Active glomerular inflammation (acute and active glomerular lesions) in various proliferative GN in humans, such as lupus nephritis, IgA nephropathy, and Henoch-Schönlein purpura nephritis, is characterized by endocapillary proliferation together with infiltrating cell accumulation and mesangiolysis, mesangial cell proliferation, necrotizing lesions with fibrin exudation, and rupture of the glomerular basement membrane with cellular crescent formation. Reiterative active glomerular inflammation is associated with the progression to chronic sclerotic glomerular lesions and the development of chronic kidney disease. ${ }^{11-15}$ Suppression of acute and active glomerular lesions, therefore, is thought to be essential to prevent the progression to end-stage renal disease (ESRD) in proliferative GN.

Thy-1 GN is a well-studied model of mesangioproliferative GN in the rat. ${ }^{16-20}$ This model is characterized by the immediate deposition of antibody on mesangial cells after antiThy 1.1 antibody injection into rats, activation of complement, and severe and diffuse immune-mediated mesangiolysis. Thereafter, focal glomerular capillary destruction, the so-called microaneurysmal ballooning, occurs in the glomeruli with fibrin exudation. Macrophage infiltration and diffuse mesangial cell proliferation develop with extracellular matrix accumulation and proteinuria. Glomerular epithelial cell injury also develops, and adherence lesions to Bowman's capsule occur in the severely damaged glomeruli. These morphologic findings in the Thy-1 GN model indicate that acute and active glomerular lesions develop with proteinuria from the early phase after disease induction.

In this study, we assessed the alteration of AT1R and AT2R expressions in glomeruli during Thy-1 GN with vehicle or ARB treatment, and examined the efficacy of ARB in the early phase of active glomerular lesions in experimental proliferative GN.

\section{MATERIALS AND METHODS Experimental Protocol}

Monoclonal antibody against rat Thy-1. 1 (OX-7; Cedarlane Laboratories, Toronto, Ontario, Canada) was administered intravenously at a dose of $60 \mu \mathrm{g} \operatorname{IgG} / 100 \mathrm{~g}$ body weight in male Wister rats. All rats weighing 180-200 g at the age of 6-7 weeks were obtained from Sankyo Labo Service Corporation (Tokyo, Japan). Thy-1 GN was induced in rats that were divided into three groups $(n=7$, in each group), Thy- 1 $\mathrm{GN}+$ high-dose (HD) ARB group (olmesartan; $3 \mathrm{mg} / \mathrm{kg} /$ day), Thy-1 GN + low-dose (LD) ARB group (olmesartan; $0.3 \mathrm{mg} / \mathrm{kg} /$ day), and vehicle (Thy-1 GN group). Seven normal rats were used as the normal control group. Rats to which HD- or LD-ARB alone had been administered were HD-ARB $(n=7)$ and LD-ARB $(n=7)$ control group, respectively. In
HD- and LD-ARB groups, administration of olmesartan was started 7 days before the induction of Thy-1 GN using an intraperitoneal micro-osmotic pump (Alzet osmotic pump, Alza, Mountain View, CA). Olmesartan (ARB), a kind gift from Daiichi Sankyo Company, Tokyo, Japan was used. BP and renal function were measured, and kidney biopsies for histological examination were performed before the implantation of the micro-osmotic pump, induction of the GN (day 0), and day 3, day 7, and day14 after Thy-1 GN induction.

\section{Systolic Blood Pressure (BP) and Renal Function}

Arterial BP was measured in the conscious state using a programmable sphygmomanometer (BP-98A) (Softron, Tokyo, Japan) by the tail-cuff method. Blood and urine samples were collected for measurements of blood urea nitrogen (BUN), serum creatinine $(\mathrm{Cr})$, urinary protein, and urine creatinine levels using an autoanalyzer (SRL, Tokyo, Japan).

\section{Histopathology and Immunohistochemistry}

For light microscopic study, the kidney tissue was fixed in $20 \%$ buffered formalin and embedded in paraffin. Tissue sections were stained with periodic acid-Schiff (PAS) for histopathological examination. For electron microscopic examination, the kidney tissue was fixed in $2.5 \%$ glutaraldehyde solution in phosphate buffer (pH 7.4) and post fixed with $1 \%$ osmium tetroxide, dehydrated and embedded in Epok 812. Ultra-thin sections were stained with uranyl acetate and lead citrate and then examined with an electron microscope (model H7100, Hitachi Corp., Tokyo, Japan).

The following primary antibodies were used for immunohistochemistry (Table 1): (1) rabbit anti-AT1R polyclonal antibody (sc-1173; Santa Cruz Biotechnology, CA, USA), which can detect the expression of rat AT1R; (2) goat anti-AT2R antibody (sc-7420; Santa Cruz Biotechnology, CA, USA), which can detect the expression of rat AT2R; (3) mouse anti-human alpha smooth muscle actin ( $\alpha \mathrm{SMA}$ ) monoclonal antibody (DAKO, Glostrup, Denmark), which is a marker for activated mesangial cells; (4) mouse anti-rat endothelial cell antigen (RECA)-1 monoclonal antibody (Serotec, Oxford, UK), which is a maker for rat endothelial cells; (5) mouse anti-rat epithelium monoclonal antibody (JG12) (BMS, Vienna, Austria), which is also used as a marker for rat endothelial cells; (6) mouse anti-rat ED-1 monoclonal antibody (BMA, Nagoya, Japan), which can detect infiltrating rat macrophages, (7) mouse anti-proliferating cell nuclear antigen (PCNA) monoclonal antibody (PC10; DAKO, Glostrup, Denmark), which is a marker for cellular proliferation; (8) mouse anti-human desmin monoclonal antibody (D33; DAKO, Glostrup, Denmark), which is a marker for injured glomerular visceral epithelial cells (podocytes); (9) rabbit anti-platelet-derived growth factor (PDGF) (Ab-1) polyclonal antibody (Oncogene Research Products, MA, USA), which can detect the expression of rat PDGF-B chain; (10) rabbit anti-NOS3 (endothelial nitric 
Table 1 Antibodies used for immunohistochemistry

\begin{tabular}{|c|c|c|c|}
\hline Recognition & Primary antibody & Dilution & Supplier/reference \\
\hline AT1R & Rabbit anti-AT1R (sc-1173) & $20(F)$ & Santa Cruz Biotechnology, CA, USA \\
\hline Activated mesangial cell & Mouse anti-human $\alpha \mathrm{SMA}$ & $50(F), 500(P)$ & DAKO, Glostrup, Denmark \\
\hline Endothelial cell & Mouse anti-RECA-1 & $20(F)$ & Serotec, Oxford, UK \\
\hline Macrophage & Mouse anti- rat ED-1 & $20(F), 250(P)$ & BMA, Nagoya, Japan \\
\hline Proliferating cell & Mouse anti-PCNA (PC10) & $100(P)$ & DAKO, Glostrup, Denmark \\
\hline Injured epithelial cell & Mouse anti-human desmin & $150(P)$ & DAKO, Glostrup, Denmark \\
\hline PDGF-B & Rabbit anti-PDGF(Ab-1) & $10(F)$ & Oncogene Research Products, MA, USA \\
\hline eNOS & Rabbit anti-NOS3 (eNOS) (sc-654) & $10(F)$ & Santa Cruz Biotechnology, CA, USA \\
\hline
\end{tabular}

AT1R, angiotensin II type 1 receptor; AT2R, angiotensin II type 2 receptor; $\alpha$ SMA, $\alpha$ smooth muscle actin; RECA-1, rat endothelial cell antigen-1; PCNA, proliferating cell nuclear antigen; PDGF, platelet-derived growth factor; eNOS, endothelial nitric oxide synthase; $F$, frozen section; $P$, paraffin section.

oxide synthesis, eNOS) polyclonal antibody (sc-654; Santa Cruz Biotechnology, CA, USA), which can detect the expression of rat eNOS; (11) rabbit anti-rat nephrin antibody, ${ }^{21}$ which is used as a marker for functional molecules of the podocyte slit diaphragm; and (12) rabbit anti-rat podoplanin antibody, ${ }^{22}$ which is used as a marker for podocytes associated with the connection between foot process and the glomerular basement membrane.

To detect AT1R, AT2R, PDGF-B, eNOS, nephrin, and podoplanin expressions in glomeruli, frozen sections were stained by the standard indirect technique. To recognize AT1R and AT2R expressions on endothelial cells, activated mesangial cells, or macrophages, double immunofluorescent staining for each receptor and RECA-1, $\alpha$ SMA, or ED-1 was performed. Briefly, $4 \mu \mathrm{m}$ frozen sections were stained with anti-AT1R antibody (rabbit IgG) or anti-AT2R antibody (goat IgG), followed by FITC-labeled goat anti-rabbit IgG antibody (ZYMED, CA, USA) or FITC-labeled donkey antigoat IgG antibody (Santa Cruz Biotechnology, CA, USA), respectively. The sections were then incubated with antiRECA- 1 or $\alpha$ SMA antibody (mouse IgG) and followed by Texas-red conjugated goat anti-mouse IgG (Leinco Technology, MI, USA). Specimens of single or double staining for detection of AT1R and AT2R were examined under a confocal scanning laser microscopy (CLSM, TCS-SP; Leica Lasertechnik, Heidelberg, Germany) based on an upright microscope (DMRB, Leica Lasertechnik) equipped with a krypton-argon laser.

To detect $\alpha$ SMA, PCNA, JG12, desmin, and ED- 1 in glomeruli, 20\%-buffered, formalin-fixed, and paraffin-embedded tissue sections were stained using Histofine Simple Stain Rat MAX PO (MULTI) (Nichirei, Tokyo, Japan) following the protocols recommended by the manufacturer. For pretreatment before incubation with the primary antibody, for JG12, $\alpha \mathrm{SMA}$, and desmin, the slides were incubated in $0.01 \mathrm{M}$ sodium citrate buffer $(\mathrm{pH} 6.0)$, at $120^{\circ} \mathrm{C}$ for $20 \mathrm{~min}$ in a steam autoclave. For ED-1, tissue sections were incubated with $0.1 \%$ pepsin $/ 0.01 \mathrm{~N} \mathrm{HCL}$ at $37^{\circ} \mathrm{C}$ for $45 \mathrm{~min}$. To identify proliferating and activated mesangial cells, double immunohistochemistry with $\alpha$ SMA and PCNA was performed using the color modification method of 3,3'-diaminobendizine (DAB) precipitation by cobalt chloride, which changes $\mathrm{DAB}$ color from brown to dark blue. ${ }^{23,24}$

\section{Real-Time PCR}

Isolated glomeruli were obtained by differential sieving through mesh brass sieves $(80,120$, and $200 \mu \mathrm{m})$. The glomerular total RNA was extracted from isolated glomeruli by Qiagen RNeasy kit (QiagenGmbH, Hilden, Germany) according to the manufacturer's instructions. A cDNA copy was created with reverse transcriptase from High Capacity cDNA Reverse Transcription kit (Applied Biosystems, CA, USA). Gene expression was analyzed by real-time quantitative RTPCR using the TaqMan system based on real-time detection of accumulated fluorescence according to the manufacturer's

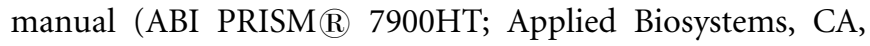
USA). To control for variations in the amount of DNA available for PCR in the different samples, gene expression of the target sequence was normalized in relation to the expression of endogenous control, 18S ribosomal RNA (TaqMan Ribosomal RNA Control kit) (Applied Biosystems, CA, USA). Primers and TaqMan probes for AT1R (Assay ID: Rn01435427_m1), AT2R (Assay ID: Rn00560677_s1), nitric oxide synthase 3 (eNOS) (Assay ID: Rn02132634_s1), PDGFB (Assay ID: Rn01502597_m1), and 18S ribosomal RNA were purchased from Applied Biosystems. 


\section{Quantification of Histopathological Findings}

In each kidney sample, more than 20 cross sections of glomeruli were examined sequentially. Quantitative and semiquantitative analyses were performed as follows: (A) Glomerular capillaries: the mean number of glomerular capillary lumina surrounded by JG12-positive cells per glomerular cross section. (B) Activated mesangial cells: the mean semiquantitative staining score of $\alpha \mathrm{SMA}$-positive area per glomerular cross section (scores $0=$ very weak or absent staining; $1+=$ up to $25 \%$ of glomeruli; $2+=25-50 \%$; $3+=50-75 \% ; 4+=>75 \%$ ). (C) Proliferating and activated mesangial cells: the mean number of both $\alpha$ SMA- and PCNA-positive cells per glomerular cross section. (D) Glomerular injured-epithelial cells: the mean semiquantitative staining score of desmin-positive glomerular edge area per total glomerular edge area (scores $0=$ very weak or absent staining; $1+=$ up to $25 \%$ of glomerular edge; $2+=25$ $50 \% ; 3+=50-75 \% ; 4+=>75 \%)$. As a reason for the assessment of glomerular edge area, desmin is a typical mesenchymal marker and is detectable in injured glomerular epithelial cells as well as activated mesangial cells in the renal glomeruli. (E) Infiltrating macrophages: the mean number of ED-1-positive cells per glomerular cross section. Glomerular cross sections containing only a small portion of the glomerular tuft were excluded from the analysis. All histopathological evaluations were performed by investigators blinded to the treatment modality.

All values are represented as mean \pm standard error (s.e.). One-way analysis of variance (ANOVA) and the Bonfferoni post hoc test were used for multiple comparisons. Statistical analysis was performed using the KaleidaGraph software. A $P$-value $<0.05$ was considered significant in all tests.

\section{RESULTS}

\section{Time Course of Systolic Blood Pressure (BP)}

After Thy-1 GN induction, systolic BP did not increase during the experiment. In normal control, LD-ARB control, and Thy-1 GN+LD-ARB groups, systolic BP was not changed significantly. In contrast, administration of HD-ARB significantly lowered systolic BP with or without Thy-1 GN induction, compared with the other groups during an experiment $(P<0.01 v s$ the other groups) (Figure 1a).

\section{Urinary Protein Excretion, Blood Urea Nitrogen, and Serum Creatinine Levels}

In the Thy-1 GN group, proteinuria developed after Thy-1 GN induction and urinary protein excretion reached a maximum from day 3 to day 7 . Both HD- and LD-ARB treated groups had significantly diminished urinary protein excretion after Thy-1 GN induction compared with the Thy-1 GN group $(P<0.01$ vs Thy-1GN group) (Figure $1 \mathrm{~b})$. Moreover, the urinary protein level of the Thy-1GN + HD-ARB group was significantly lower than that of the Thy-1 GN + LD-ARB group $(P<0.05$ vs Thy-1 GN + LD-ARB group). On the other hand, BUN and serum $\mathrm{Cr}$ levels in the
Thy-1 GN group did not change during the course of the disease. No significant differences of BUN and serum Cr levels were observed between all groups. In HD- and LD-ARB control groups, urinary protein excretion, BUN and serum Cr levels did not change significantly after ARB administration.

\section{Alteration of Angiotensin II Type I Receptor (AT1R) and Type II Receptor (AT2R) Expression in Glomeruli}

The glomerular expression of AT1R and AT2R, as determined by immunofluorescence, are shown in Figure 2. The glomerular expression of AT1R and AT2R were weak in three control groups (HD-ARB, LD-ARB, and normal control groups). On day 3, in both ARB-treated and Thy-1GN groups, the AT1R expression was slightly decreased. On day 7, the AT1R expression was augmented transiently in the Thy-1 GN group, however, administration of ARB inhibited the enhancement of AT1R expression. Conversely, the expression of AT2R in both ARB-treated and Thy-1 GN groups was increased on day 3. ARB treatment mediated a further increase in the AT2R expression from day 3 to day 14 . The mRNA level of AT1R and AT2R in isolated glomeruli was assessed by real-time PCR (Figure 2). Administration of HDor LD-ARB alone did not significantly change the glomerular mRNA expression of both receptors during an experiment compared with normal controls. On day 3 , in both ARBtreated and Thy-1 GN groups, AT1R expression was reduced transiently by Thy-1 GN induction compared with all control groups. On day 7, the AT1R expression in the Thy-1 GN group was obviously enhanced. This reaction was suppressed by the administration of HD-ARB $(P<0.01$ vs Thyl GN or Thy-1 GN + LD-ARB group). On the other hand, the expression of AT2R in glomeruli was upregulated by disease induction. Furthermore, this response was augmented significantly by ARB treatment from day 3 to day14. The enhancement effect on the AT2R expression showed ARB dose dependency.

We also examined the origin of cells that expressed AT1R and AT2R in glomeruli (Figure 3). In double immunofluorescence studies of ARB-treated groups and the Thy-1 GN group, AT1R and AT2R were detected on $\alpha$ SMA-positive activated mesangial cells and RECA-1-positive glomerular endothelial cells on day 7. In AT1R and RECA-1, or AT2R and RECA-1-positive cells located on the other side of the glomerular basement membrane, a large angiotensin II receptor-positive area was seen, implying that both the receptors were also present in the glomerular epithelial cells. In addition, AT1R and AT2R were detected on ED-1-positive macrophages in glomeruli. It seemed that the degree of both the receptors' expression in each cell was slightly different between ARB-treated groups and the Thy-1 GN group. In ARB-treated groups, cells double positive for expressions of AT1R, and RECA-1 or $\alpha$ SMA were confined to a small area as compared with the Thy-1 GN group. Conversely, double- 

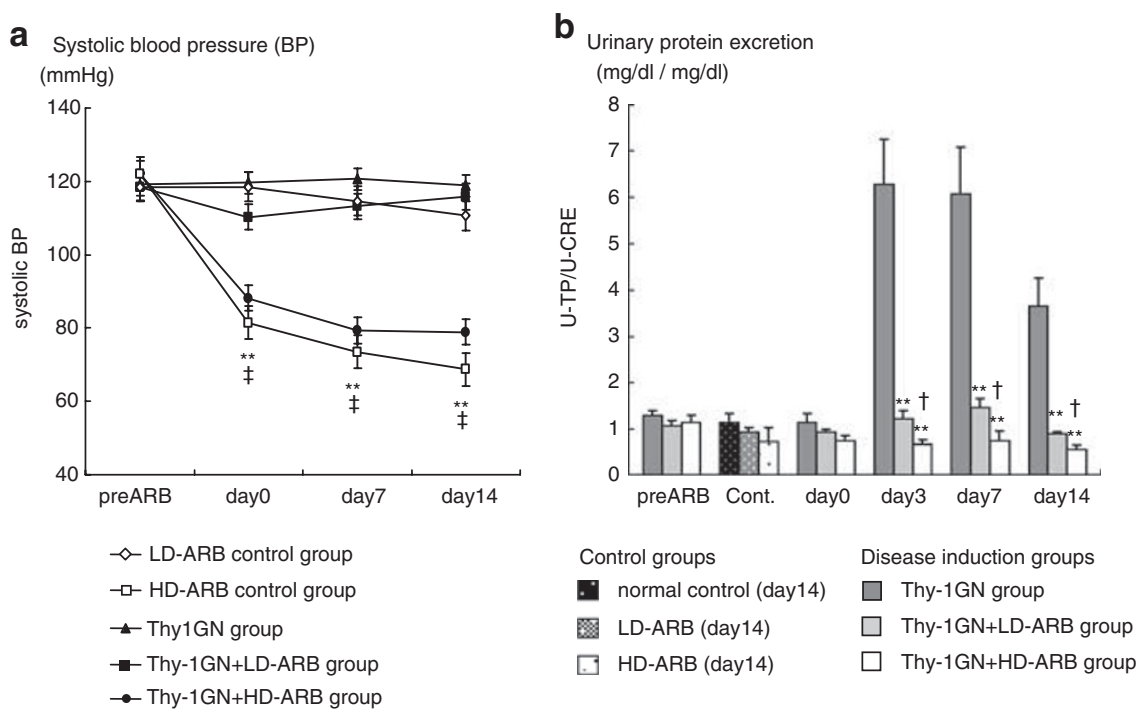

Figure 1 Systolic blood pressure (BP) (a) and urinary protein excretion (b) in the Thy-1 GN, Thy-1 GN + high dose (HD)-ARB, Thy-1 GN + low dose (LD)-ARB, normal control, and ARB control groups. Systolic BP was significantly lower in the Thy- 1 GN + HD-ARB and HD-ARB control groups when compared with that of the other groups. Urinary protein excretion was inhibited by HD- and LD-ARB treatments. Each column plot denotes the mean \pm s.e. ${ }^{*} P<0.01$ vs Thy$1 \mathrm{GN},{ }^{\dagger} P<0.05$ vs Thy-1GN + LD-ARB group, ${ }^{\ddagger} P<0.01$ vs Thy-1GN + LD-ARB group.

positive areas of AT2R, and RECA-1 or $\alpha$ SMA were widely seen than the Thy-1 GN group.

\section{The Effects of ARB on Acute and Active Glomerular Lesions}

After disease induction, diffuse mesangiolysis occurred by day 3 in all disease induction groups (Figure 4). This indicates that ARB did not affect antibody and complementmediated mesangial cell-lysis in Thy-1 GN. Thereafter, capillary destruction and aneurysmal ballooning lesions were observed with fibrin exudation in the Thy-1 GN group. However, these lesions were not prominent in ARB-treated groups. From day 7 to day 14, marked mesangial cell proliferation developed in the Thy-1 GN group, whereas mild and segmental proliferative lesions were observed in ARBtreated groups. In addition, focal adhesion to Bowman's capsule was observed partly, and injured glomeruli progressed to focal segmental sclerosis in the Thy-1 GN group. In contrast, in ARB-treated groups, damaged glomeruli showed an almost normal glomerular structure with only mild proliferative lesions by day 14 .

Next, we assessed the effects of ARB on each active glomerular lesion after induction of Thy-1 GN. First, we checked the effects of ARB on glomerular endothelial injury and the subsequent capillary destruction. In the Thy-1 GN group on day 7, JG12-positive capillary lumens disappeared in ballooning lesions, indicating that destruction of the glomerular capillary network developed with a loss of glomerular endothelial cells (Figure 5). On the other hand, in ARB-treated groups, JG12-positive capillary loops were comparatively maintained on day 7. Quantitative analysis of JG12-positive capillary lumens showed ARB treatment significantly prevented the loss of glomerular capillary loops from day 3 to day 7 ( $P<0.01 v s$ Thy-1 GN group). We also ascertained the decrease of the glomerular expression of eNOS as a marker for endothelial dysfunction. The expression of eNOS in normal glomeruli was located on glomerular capillaries. In the Thy-1 GN group, the expression of eNOS was diminished in ballooning and proliferative lesions on day 7. However, in the Thyl-GN + HD-ARB group, eNOS expression was maintained at similar levels to all control groups. The retention of glomerular mRNA expression of eNOS was also observed on day $7(P<0.05$ vs Thy-1 GN group, $P<0.01$ vs LD-ARB group). Electron microscopic findings revealed swelling of the endothelial cells with loss of fenestra in the Thy-1 GN group, whereas endothelial activation diminished with preserved fenestra in ARB-treated groups (Figure 6).

In mesangial proliferative lesions, the $\alpha \mathrm{SMA}$ staining score in both ARB treated groups was significantly lower than in the Thyl-GN group on day 7 and day 14. (day 7; $P<0.01$ Thy-1 GN + HD-ARB group $v$ shy-1 GN group, $P<0.05$ Thy-1 GN + LD-ARB group vs Thy-1 GN group, day14; $P<0.01$ Thy-1 GN + HD-ARB group vs Thy-1 GN group). The number of glomerular cells positive for both $\alpha$ SMA and PCNA was also clearly lower in both the HD- and the LDARB treated rats on day $7(P<0.01$ vs Thy-1 GN group) (Figure 7). A notable expression of PDGF-B was observed in the Thy-1 GN group on day 7, whereas the expression of PDGF-B was minimal in ARB-treated groups as well as all control groups. The glomerular mRNA expression of PDGFB was depressed in ARB-treated groups on day $3(P<0.05 v s$ Thy-1 GN group). In electron microscopic findings, swelling 

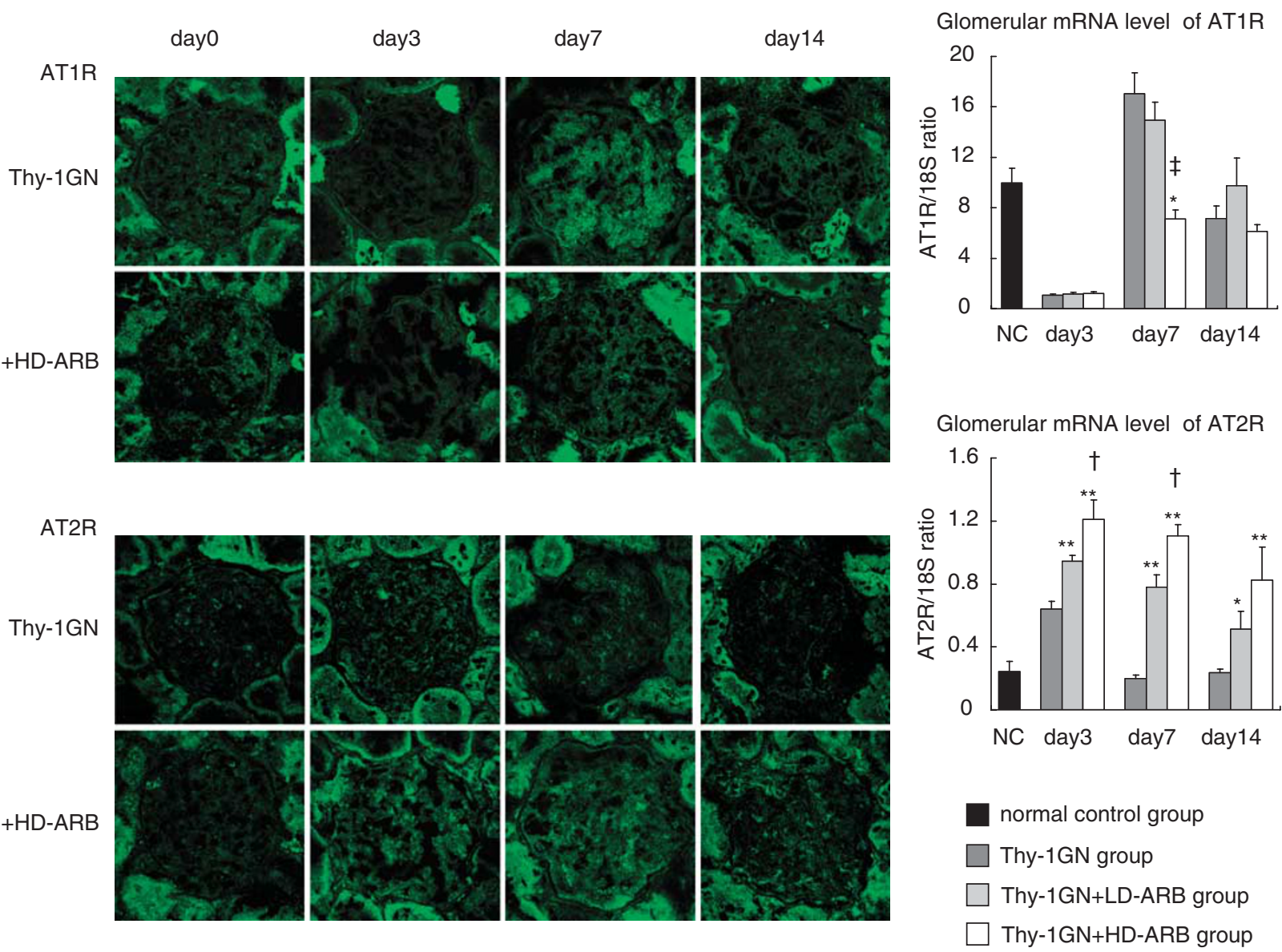

Figure 2 The expression of angiotensin II type 1 receptor (AT1R) and type 2 receptor (AT2R) in glomeruli in normal controls, Thy-1 GN and HD-ARB treated groups. AT1R and AT2R: in immunofluorescence studies, the glomerular expressions of both AT1R and AT2R were weak in the normal glomeruli. The similar staining pattern was observed in ARB-treated groups on day 0 before disease induction. On day 3, in ARB-treated and the Thy- 1 GN groups, the AT1R expression was slightly decreased by Thy- $1 \mathrm{GN}$ induction. Conversely, the AT2R expression was increased between groups. In addition, the staining of AT2R in the ARB-treated group was more intense than that in the Thy-1 GN group. On day 7, the staining of AT1R in the Thy-1 GN group was augmented transiently. ARB treatment inhibited the enhancement of the AT1R expression. The expression of AT2R was increased by Thy-1 GN induction and further augmented by ARB treatment. The glomerular mRNA levels of AT1R and AT2R: by real-time PCR, on day 3, the glomerular mRNA expression of AT1R was reduced by disease induction. On day 7, the AT1R expression in the Thy-1 GN group was increased transiently than the normal control group.

Administration of HD-ARB suppressed this reaction prominently. The expression of $A T 2 R$ was upregulated by disease induction. Furthermore, this effect was augmented significantly by ARB treatment. Each column plot denotes the mean \pm s.e. ${ }^{* *} P<0.01$ vs Thy $-1 \mathrm{GN},{ }^{\dagger} P<0.05$ vs Thy- $1 \mathrm{GN}+\mathrm{LD}-\mathrm{ARB}$ group, ${ }^{\ddagger} P<0.01$ vs Thy-1GN + LD-ARB group.

of mesangial cells was noted with large nuclei and formation of mesangial interposition around glomerular capillaries in the Thy-1 GN group. In contrast, mesangial cell swelling diminished with no mesangial interposition in ARB-treated groups (Figure 6).

Analysis of glomerular epithelial damage revealed that a desmin-positive area, representing injured epithelial cells, appeared strongly in the Thy-1 GN group (Figure 8). In ARB-treated groups, desmin staining was close to baseline levels. Consistent with this finding, the score index for desmin-positive epithelial cells was significantly lower in ARBtreated groups (day 7; both $P<0.01 v s$ Thy-1 GN group, day 14; both $P<0.05$ vs Thy-1 GN group). To determine whether glomerular epithelial cells were maintained functionally in
ARB-treated groups, we examined the expressions of a slitmembrane molecule (nephrin) and a cell surface molecule (podoplanin) on epithelial cells. The expressions of nephrin and podoplanin in normal glomeruli were observed clearly and continuously on epithelial cells. However, in Thy-1 GN, the expression of both molecules were weak and discontinuous. On the other hand, ARB treatment kept nephrin and podoplanin expressions at near normal levels. Electron microscopic findings showed swelling of glomerular epithelial cells with effacement of foot processes in the Thy-1 GN group, whereas this epithelial cell injury was less severe with intact foot process in ARB-treated groups (Figure 6).

The number of glomerular ED-1-positive macrophages was significantly less in ARB-treated groups than in the 
a
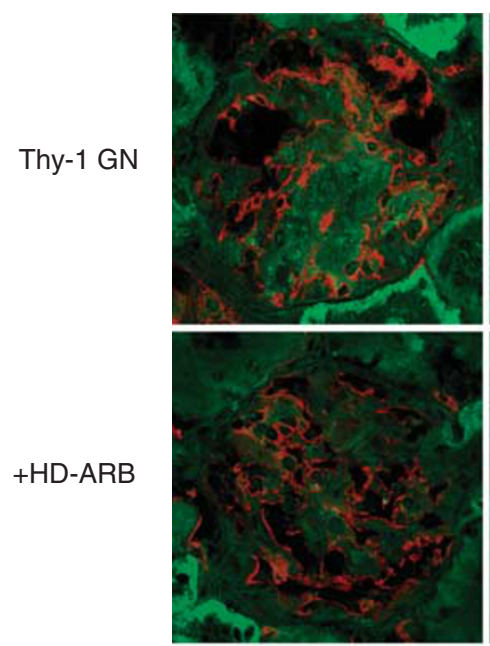

b

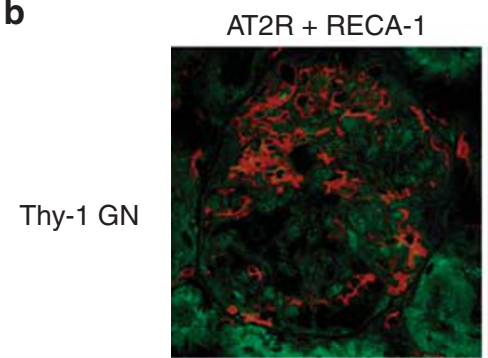

+ HD-ARB

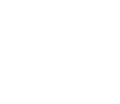

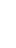

+HD-ARB

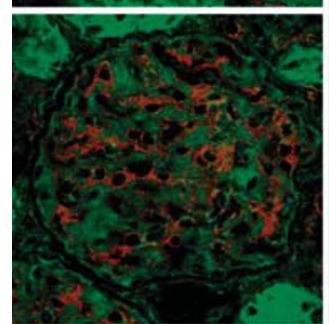

$A T 1 R+\alpha S M A$
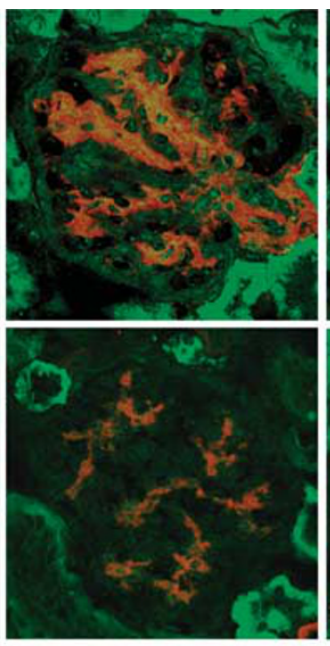

$\mathrm{AT} 2 \mathrm{R}+\alpha \mathrm{SMA}$
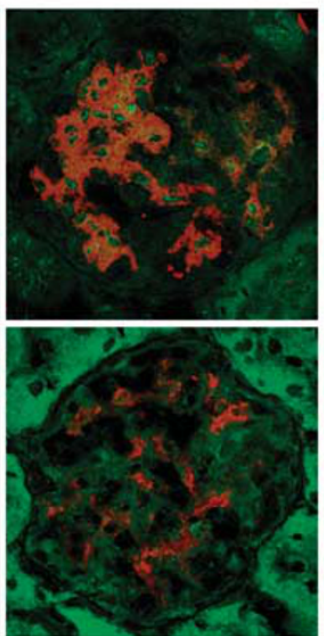

$A T 1 R+E D-1$

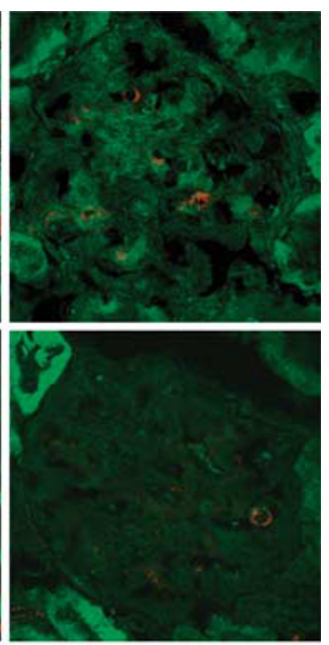

AT2R + ED-1
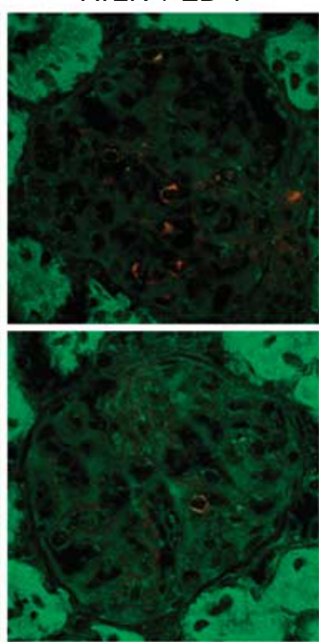

Figure 3 The expression of angiotoensin II type 1 receptor (AT1R) (a) and type 2 receptor (AT2R) (b) on the glomerular endothelial cells, activated mesangial cells, and infiltrating macrophages in ARB-treated groups and the Thy-1 GN group on day 7. In part, each staining of AT1R (green) and AT2R (green) was observed on RECA-1-positive glomerular endothelial cells (red), on $\alpha$ SMA-positive activated mesangial cells (red), and on ED-1-positive infiltrating macrophages (red). The expressions of AT1R and AT2R on glomerular epithelial cells were detected as both receptors expression in the other side of the glomerular basement membrane from AT1R + RECA-1 or AT2R + RECA-1-positive cells.

Thy-1 GN group, showing that ARB treatment abrogated macrophage accumulation into the glomeruli (Figure 9).

\section{DISCUSSION}

This study showed that the changes of angiotensin II receptors' expression in glomeruli during experimental mesangioproliferative GN treated with vehicle or ARB, and the powerful actions of ARB on acute destructive glomerular injuries. We confirmed that angiotensin II receptors (AT1R and AT2R) were expressed on glomerular endothelial, mesangial, epithelial cells, and macrophages. ARB mediates the downregulation of AT1R and the upregulation of AT2R. That may be one of the mechanisms for renal protection by ARB. In addition, $A R B$ inhibits the acute destructive and proliferative glomerular lesions, through the prevention of glomerular endothelial, mesangial, and epithelial injuries, and the reduction of macrophage infiltration in Thy-1 GN. These findings indicate ARB may affect individual glomerular cells and macrophages through angiotensin II receptors, with the alteration of both AT1R and AT2R expressions.

RAS plays a key role in the maintenance of normal BP and organ function. In the RAS, angiotensin II is the major bioactive peptide whose function is mediated by two specific receptors, AT1R and AT2R. Apart from its systemic hemodynamic effects, angiotensin II promotes the production of pro-inflammatory mediators, cell proliferation, extracellular matrix synthesis, and release of reactive oxygen species. ${ }^{2,4,5}$ 


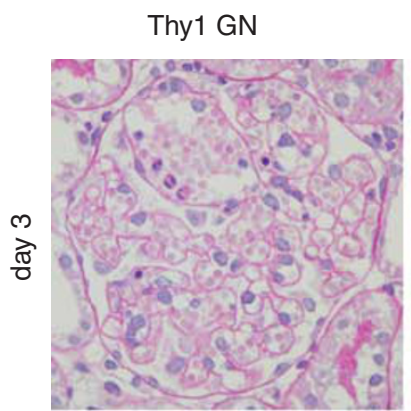

Thy $1 \mathrm{GN}+\mathrm{HD}-\mathrm{ARB}$
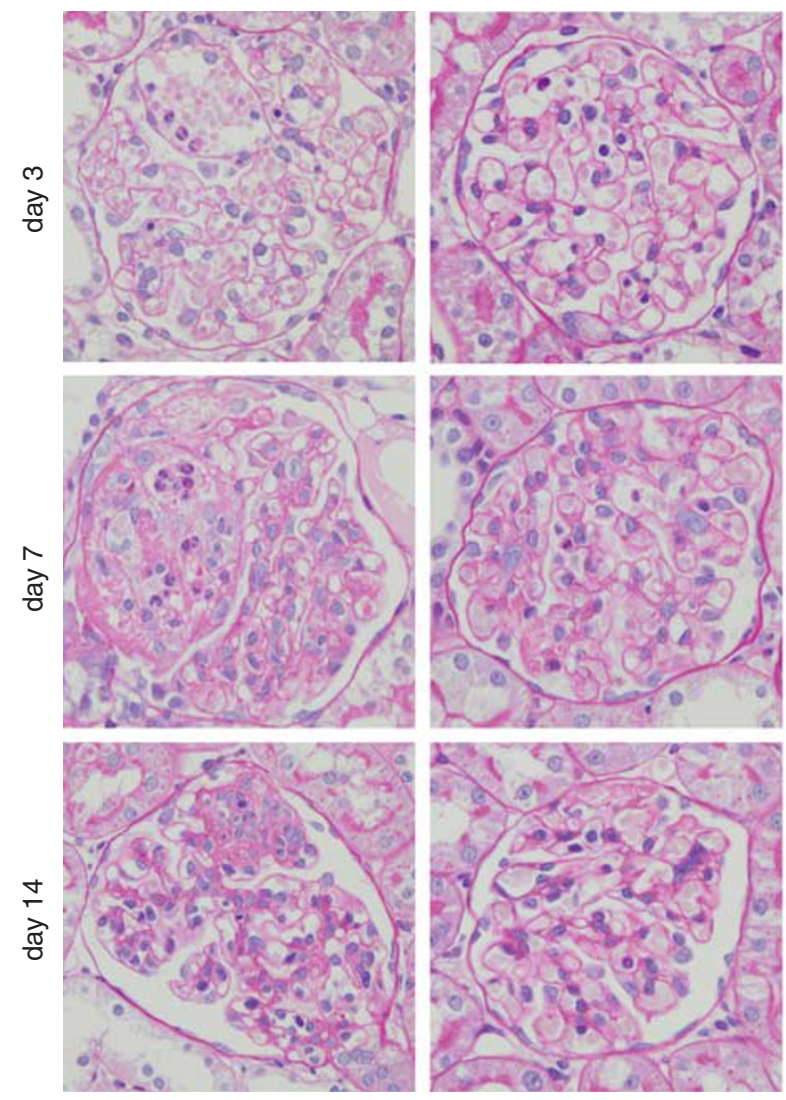
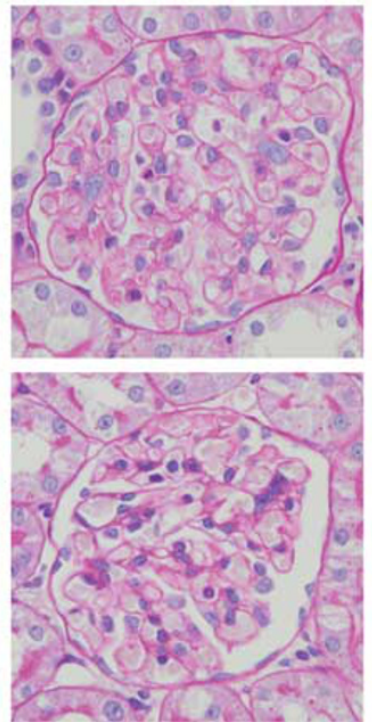

Thy-1GN+LD-ARB
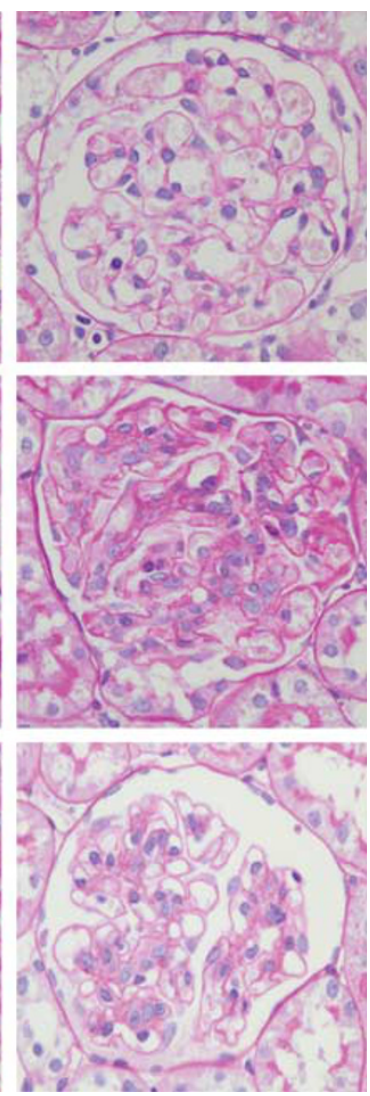

Figure 4 Diffuse mesangiolysis, aneurysmal ballooning, and proliferative lesions in the Thy-1 GN, Thy-1 GN + HD-ARB, and Thy-1 GN + LD-ARB groups on day 3, day 7, and day 14 (PAS stain). In all disease induction groups, diffuse mesangiolysis occurred by day 3. Aneurysmal ballooning lesions were observed in the Thy-1 GN group; however, these lesions were not prominent in ARB-treated groups. On day 7, mild and segmental proliferative lesions occurred in ARB-treated groups, although marked mesangial cell proliferation developed in the Thy-1 GN group. On day 14, segmental proliferative lesions persisted with focal adhesion to Bowman's capsule in the Thy-1 GN group. In contrast, in ARB-treated groups, damaged glomeruli recovered to an almost normal glomerular structure.

AT1R is responsible for most of the pathophysiological actions of angiotensin II, and inappropriate activation of the angiotensin II-AT1R pathway mediates the development of chronic kidney disease and the progression to ESRD. Moreover, it is thought that the angiotensin II-AT2R pathway, resulting in vasodilatation, decreased renal sodium re-absorption, anti-inflammation, and inhibition of cell growth, antagonizes the AT1R signaling, and may prevent the progression of renal diseases. ${ }^{25-27}$ Recently, it has been established that angiotensin II is not only generated in the circulation by renin and ACE but is also produced locally in various organs including kidney, vessels, heart, and brain. ${ }^{28,29}$ Kidney damage through the angiotensin II through AT1R activation is induced by local angiotensin II rather than by circulating angiotensin II. In this study, we did not measure the circulating angiotensin II level, because it is known that the concentration of tissue angiotensin II does not correlate to circulating angiotensin II level.

Both the AT1 and AT2 receptors are expressed in many cardiovascular and other tissues. Several reports have shown that both the receptors are expressed in the kidneys, and the expression of these receptors is altered in glomerular diseases in humans and experimental models. ${ }^{27,30-35}$ However, the alteration of AT1R and AT2R in glomeruli was examined mainly in diabetic nephropathy, and only a few were reported in proliferative GN that showed the reduction of AT1R expression in the whole kidney or microdissected glomeruli in humans. ${ }^{33}$ In this study, we confirmed the expressions of both the angiotensin II receptors in glomerular endothelial, mesangial, and epithelial cells and infiltrating macrophages, and revealed that the glomerular expressions of both AT1R and AT2R were altered by Thy-1 GN induction. On day 7, both AT1R and AT2R expressions in glomeruli were increased by disease induction. Importantly, ARB treatment suppressed the augmentation of AT1R expression. On the other hand, AT2R upregulation was further enhanced by ARB treatment. These results suggest that the AT1R expression in glomeruli is augmented in acute glomerular lesions, and that may accelerate glomerular inflammation and injuries by angiotensin II. ARB may exert renoprotective effects through both the 

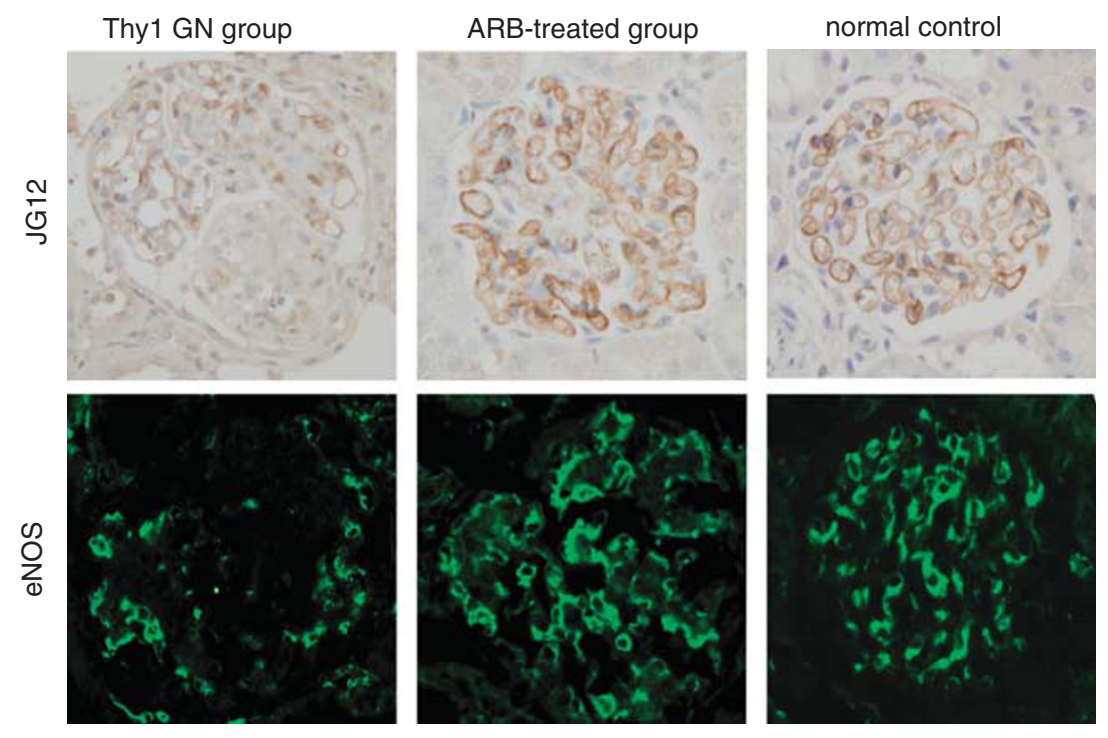

JG12(+) glomerular capillaries

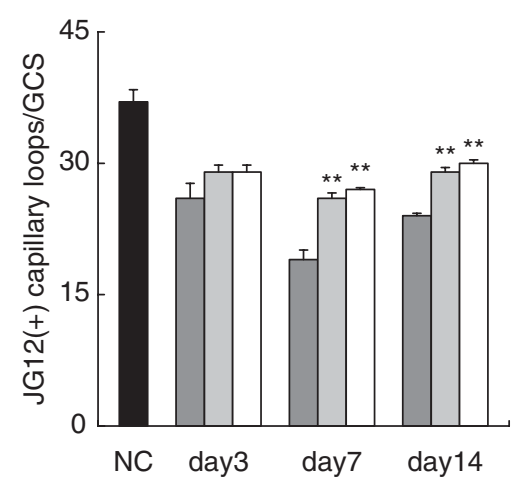

normal control group

Thy-1GN group
The glomerular mRNA level of eNOS

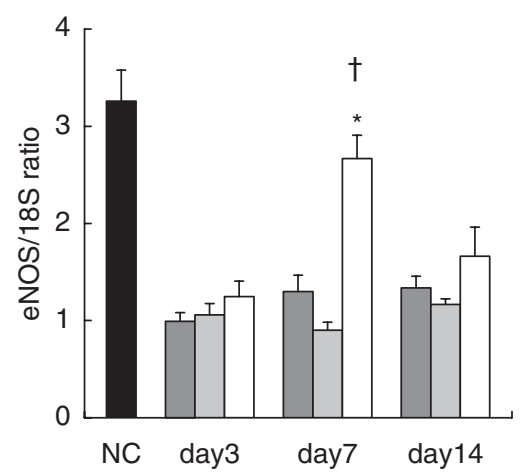

Thy-1GN+LD-ARB group

Thy-1GN+HD-ARB group

Figure 5 Capillary network and eNOS expression in glomeruli in Thy-1 GN, ARB-treated, and normal control groups. JG12: in the Thy-1 GN group, JG12positive glomerular endothelial cells disappeared in ballooning lesions and proliferative lesions on day 7. In contrast, JG12-positive capillary loops were maintained in ARB-treated groups. eNOS: the staining of eNOS in normal glomeruli was found mainly in glomerular capillary loops. In the Thy1 GN group, eNOS-positive glomerular capillary loops diminished in ballooning and proliferative lesions. However, in the Thy-1 GN + HD-ARB group, eNOS expression was retained on glomerular capillary network. The number of JG12-positive capillary lumens per glomerular cross section (GCS): ARB treatment prevented significantly the loss of glomerular capillary loops from day 7 to day 14. The mRNA level of eNOS in isolated glomeruli by real-time PCR: the retention of glomerular mRNA of eNOS on day 7 was observed in the Thy-1 GN + HD-ARB group. Each column plot denotes the mean \pm s.e. ${ }^{\star} P<0.05$ vs Thy-1 GN group, ${ }^{*} P<0.01$ vs Thy- $1 \mathrm{GN}$ group, ${ }^{\dagger} P<0.05$ vs Thy- $1 \mathrm{GN}+$ LD-ARB group.

inhibition of AT1R activation and the enhancement of AT2R action.

So far, it has been shown that ARB treatment has beneficial roles in mainly chronic kidney disease. ${ }^{10}$ ARB reduces chronic glomerular capillary hypertension and hyperfiltration, which leads to mesangial matrix expansion, glomerular sclerosis, and tubulointerstitial damage. ARB also has renoprotective advantages, being independent of the effects on systemic hypertension, and including the inhibition of cell proliferation and collagen deposition because of the decreased expression of profibrotic cytokines and growth fac- tors. $^{3,4,6}$ The renoprotective effects of ARB have been shown using various experimental models of chronic kidney disease and progressive interstitial fibrosis. ${ }^{8,9,36-39}$ In addition, it is also suggested that ARB contributes to the regression of chronic kidney disease. ${ }^{8,9,40}$ Some studies have reported beneficial effects of ARB using the Thy-1 GN model, and some of the studies used a progression model with uninephrectomy. ${ }^{41-49}$ Most of these reports presented the inhibition of macrophage infiltration, mesangial cell proliferation, and the progression of glomerular sclerosis through the suppression of the profibrotic growth factor. 


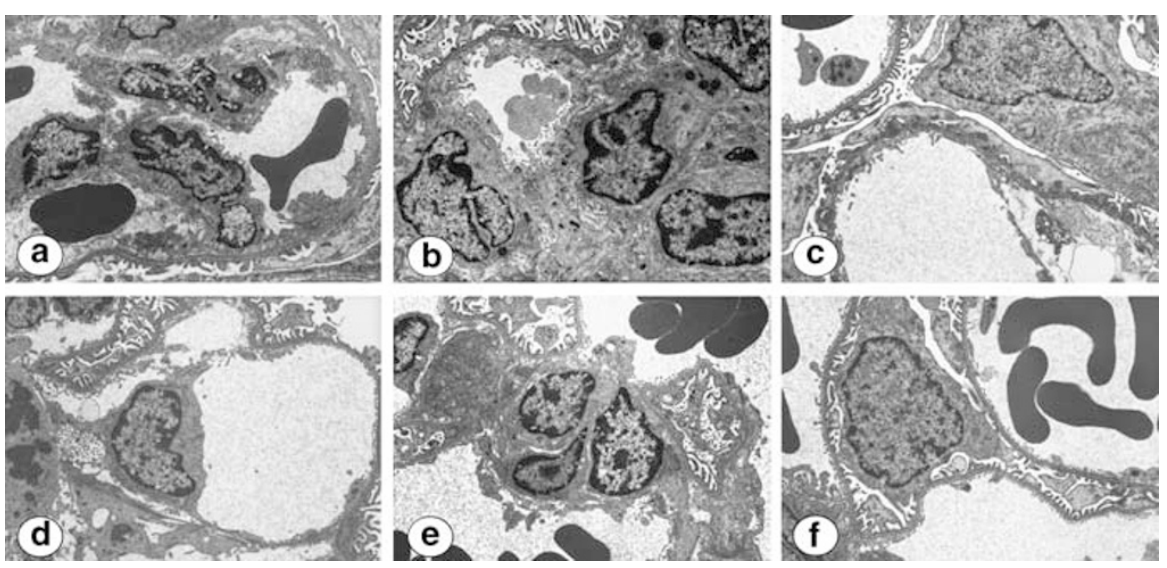

Figure 6 Glomerular endothelial (a, d), mesangial (b, e), and epithelial (c, f) cells in the Thy-1 GN group (a-c), and the Thy-1 GN + HD-ARB group (d-f) on day 7 ( $\times 4000)$. In endothelial cells, swelling and loss of fenestra was seen in the Thy-1 GN group, but these alterations were prevented in the ARB-treated group, although mesangiolysis (loss of mesangial cells and mesangial matrix) developed around these capillary networks in both groups. In mesangial proliferative lesions, proliferating mesangial cells in the Thy-1 GN group were characterized by enlarged cells with large nuclei suggesting activated mesangial cells. Mesangial interposition was also detected around capillaries. Mesangial matrix began to be accumulated around mesangial cells. However, mesangial cells in mesangial proliferative lesions in the ARB-treated group had small nuclei. Mesangial matrix-lysis still continued around mesangial cells in the ARB-treated group. These findings indicated that the activation of mesangial cells was prevented by ARB treatment. In epithelial cells, enlarged cells were evident with foot process effacement in the Thy-1 GN group, suggesting that epithelial cell damage developed on day 7 . In contrast, glomerular epithelial cells had intact foot process on the glomerular basement membrane, suggesting that epithelial cell damage was prevented in the Thy-1 GN + HD-ARB group.

However, the renoprotective effects of ARB on acute glomerular lesions, in proliferative GN, have not been presented in detail. In this study, we showed that ARB treatment preserved the glomerular capillary network from destructive injuries with fibrin exudation, suppressed mesangial cell proliferation and activation, and reduced podocyte injury and the prevention of adherence to Bowman's capsule in Thy-1 GN. These protective effects on acute and active glomerular injuries may inhibit the progression of GN, which leads to ESRD, as prolonged or repeated glomerular injuries promote the development of glomerular sclerosis and the progression of chronic kidney disease.

Moreover, we revealed protective effects of ARB on macrophage infiltration. An earlier in vitro study presented that RAS components existed on macrophages and that angiotensin II facilitated differentiation of monocyte into macrophage. ${ }^{50} \mathrm{ED}-1$-positive macrophages also express AT1R and AT2R in vivo. There are two possibilities for the finding decreased macrophages in the glomeruli, either ARB suppressed macrophage infiltration into the glomeruli or suppressed monocyte differentiation into macrophages. ARB may inhibit macrophage accumulation in damaged glomeruli and reduce subsequent glomerular inflammation.

ARB has distinct local effects in the kidney in addition to its systemic hemodynamic effects. ${ }^{46,51}$ Our data showed that LD-ARB treatment significantly reduced urinary protein excretion, and inhibited acute and active glomerular lesions of experimental Thy-1 GN without lowering BP. Furthermore, in this study, the HD-ARB group rats had superior effects on urinary protein excretion and prevention of acute and active glomerular lesions to the LD-ARB-treated rats. These data indicate that ARB has powerful renoprotective effects in the HD-ARB-treated rats mediated through the dose-dependent direct effects of ARB on glomerular injuries, as well as the prevention of systemic and glomerular hypertension. It is thought that the BP-lowering effect by HD-ARB was not only regulated by the kidney, but also by the effect on other vessels. Local RAS system exists in heart and systemic vasculatures, and ARB also exerts protective effects on the progression of cardiovascular diseases, and inhibits atherosclerosis in chronic kidney disease. ${ }^{52,53}$ In this study, it is necessary to consider the renoprotection by ARB through the beneficial effects on cardiovasculature.

Recently, it has been revealed that RAS plays an essential role for the regulation of embryonic stem cells in hematopoiesis. ${ }^{54}$ Both the angiotensin II receptors are expressed on hematopoietic and endothelial progenitor cells. The inhibition of AT1R and the augmentation of AT2R have been reported to favor hematopoietic and endothelial progenitor expansion. On the other hand, the expression of Thy-1.1 has also been shown on stem cells and progenitor cells in addition to the high levels of Thy 1.1 expression in the brain, thymus, and renal glomeruli in rats. ${ }^{55,56}$ Circulating antiThy-1.1 antibody can react with the tissues under specific conditions such as high Thy-1.1 expression and the absence of endothelial barriers. ${ }^{16}$ Administered anti-Thy-1.1 antibody, therefore, may react with stem cells and progenitor cells as well as glomerular mesangial cells, thymocytes, and lymphocytes. In our study, there is a possibility that lymphocytes, stem cells, and progenitor cells were depressed by reaction 

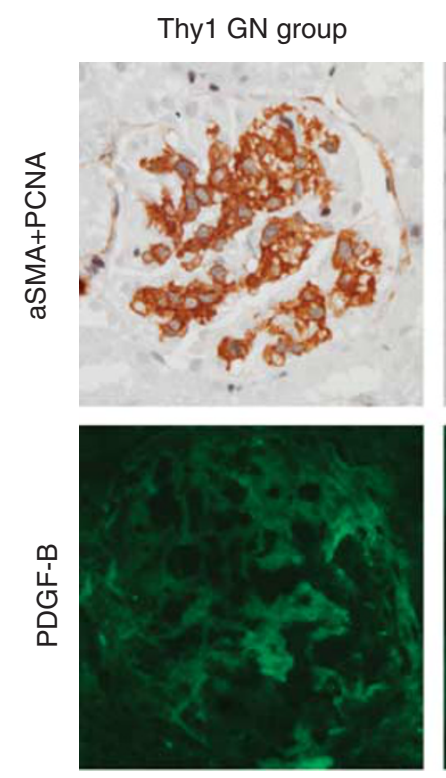

Activated mesangial cells

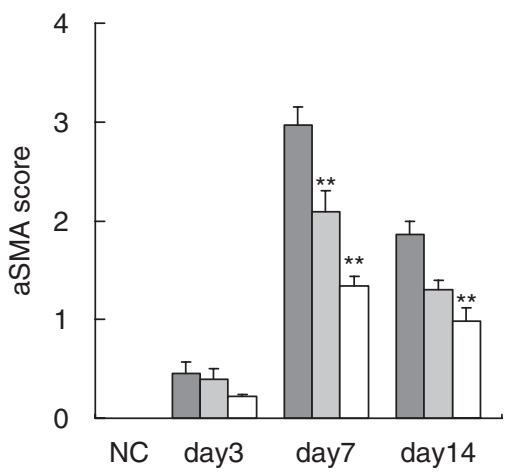

Glomerular mRNA level of PDGF-B

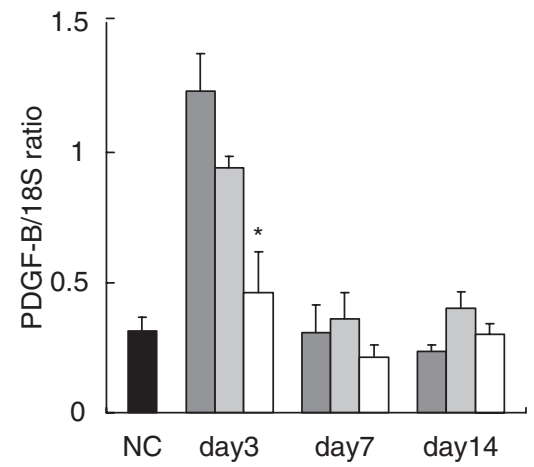

ARB-treated group

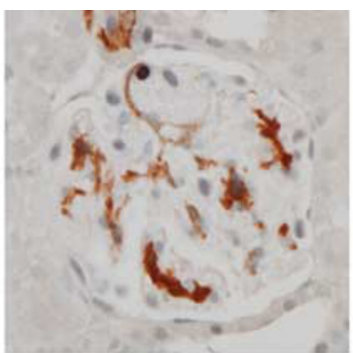

normal control
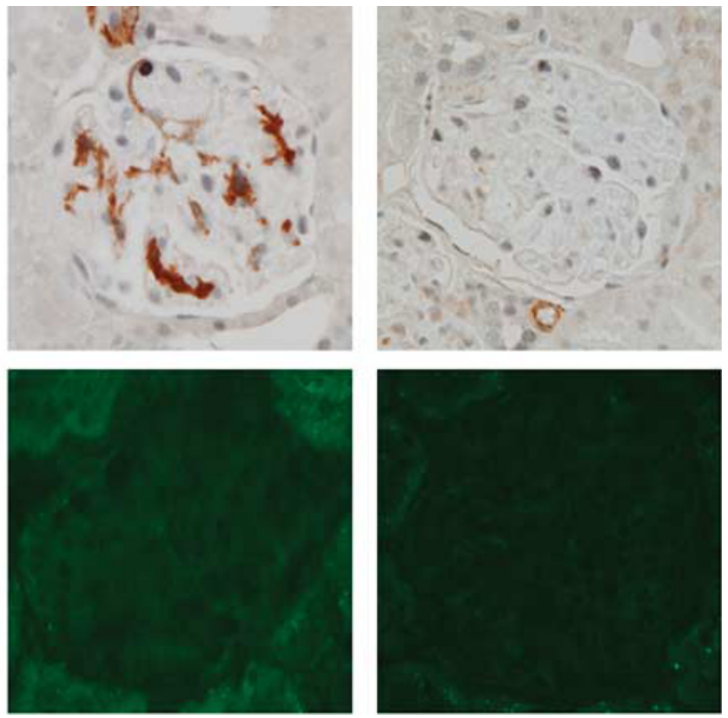

Proliferating activated mesangial cells

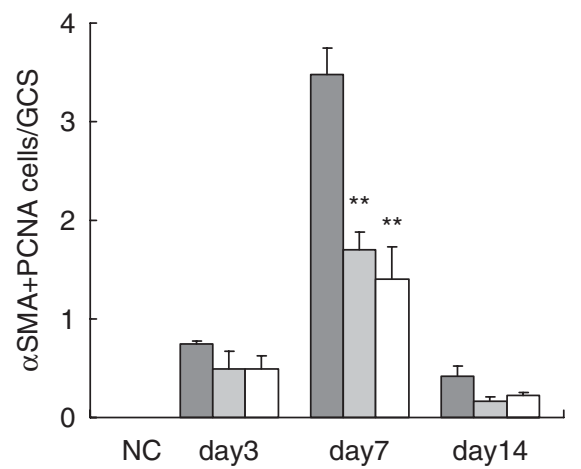

Figure 7 Proliferating and activated mesangial cells ( $\alpha$ SMA, $\alpha$ SMA + PCNA) and the expression of PDGF-B in Thy-1 GN, ARB-treated, and normal control groups. Double stain with $\alpha$ SMA + PCNA: the glomerular $\alpha$ SMA + PCNA-positive proliferating and activated mesangial cells were clearly diminished by ARB treatment on day 7. PDGF-B: the glomerular expression of PDGF-B was increased in damaged glomeruli in the Thy-1 GN group on day 7; however, ARB treatment inhibited the enhancement of PDGF-B expression by induction of Thy- $1 \mathrm{GN}$. The staining score of $\alpha$ SMA-positive activated mesangial cells per glomerular cross section (GCS): ARB significantly inhibited the mesangial activation on day 7 and day 14. The number of $\alpha$ SMA + PCNA-positive proliferating and activated mesangial cells per GCS: ARB treatment significantly inhibited the proliferation and activation of mesangial cells on day 7. The mRNA levels by real-time PCR of PDGF-B in isolated glomeruli: the glomerular mRNA of PDGF-B increased in the Thy-1 GN group on day 3. However, the administration of ARB suppressed PDGF-B production in the Thy- $1 \mathrm{GN}+$ HD-ARB group. Each column plot denotes the mean \pm s.e. ${ }^{\star} P<0.05$ vs Thy $1 \mathrm{GN}$ group, ${ }^{* * P}<0.01$ vs Thy1GN group. 


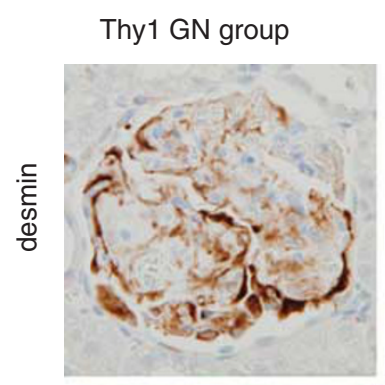

ARB-treated group
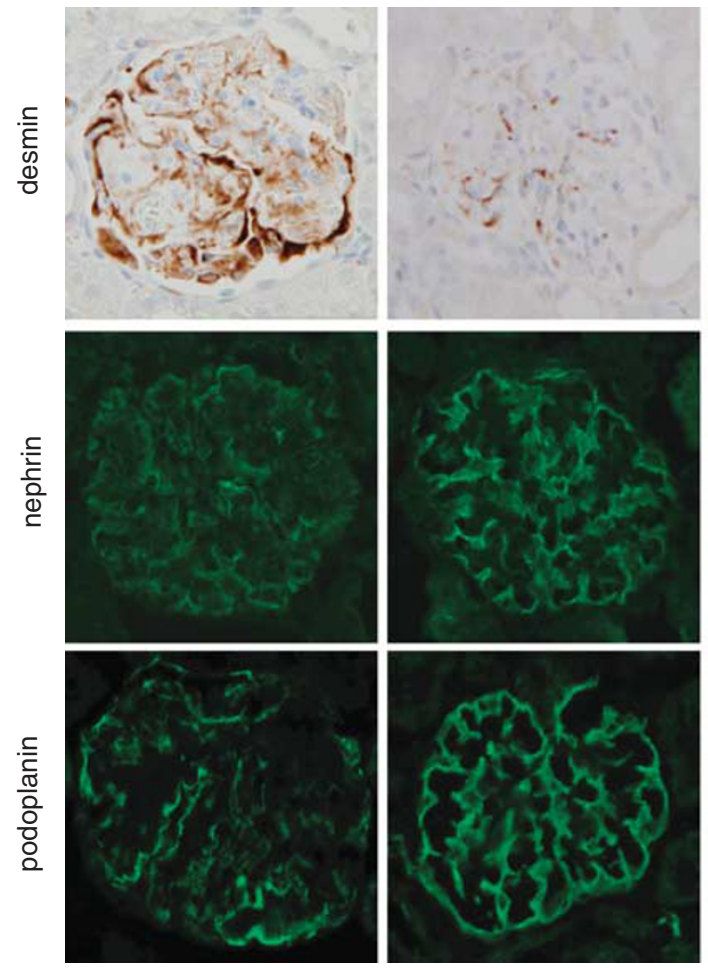

normal control
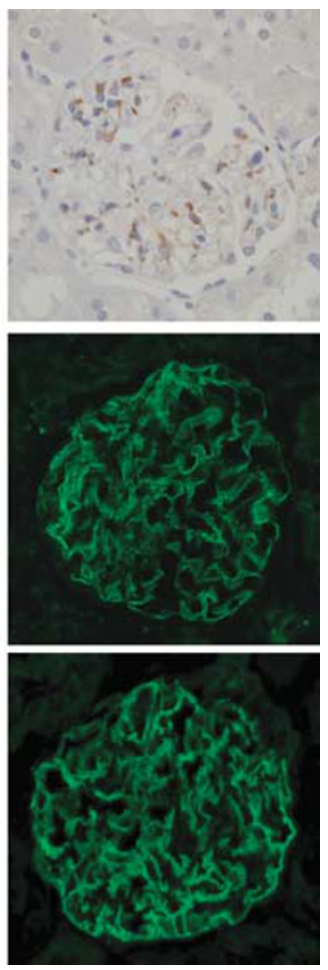

Injured glomerular epithelial cells

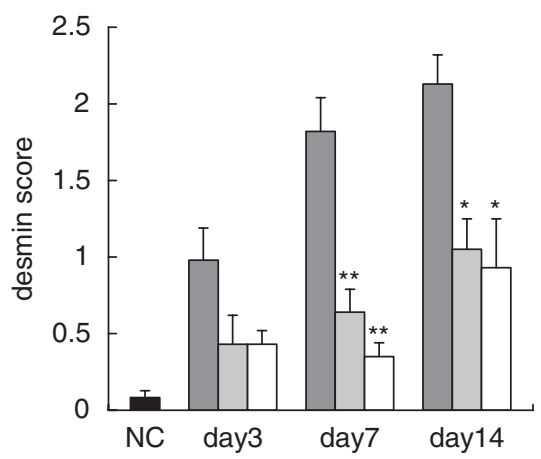

normal control group

Thy-1GN group

Thy-1GN+LD-ARB group

Thy-1GN+HD-ARB group

Figure 8 Glomerular epithelial cell injury (desmin) and the expression of nephrin and podoplanin in Thy-1 GN, ARB-treated, and normal control groups. Desmin: desmin-positive area at epithelial cells (damaged glomerular epithelial cells) strongly appeared in the Thy 1 GN group on day 7 . In ARB-treated groups, desmin staining was at nearly normal levels. nephrin-podoplanin: the expression of nephrin and podoplanin in normal glomeruli were observed clearly and continuously on glomerular epithelial cells. However, in Thy $1 \mathrm{GN}$, the nephrin and podoplanin expression were weak and discontinuous. On the other hand, ARB treatment kept the nephrin and podoplanin expression near baseline levels on day 7. The staining score of desmin-positive damaged epithelial cells in the glomerular cross section: ARB significantly inhibited the glomerular epithelial cell injury on day 7 and day 14 . Each column plot denotes the mean \pm s.e. ${ }^{\star} P<0.05$ vs Thy $1 \mathrm{GN}$ group, ${ }^{* *} P<0.01$ vs Thy $1 \mathrm{GN}$ group.

against anti-Thy-1.1 antibody. In several forms of renal disease, endothelial progenitor cells from bone marrow are available for recruitment into injured tissues and facilitate endothelial cell repair. ${ }^{57}$ In the Thy-1 GN model, it has been documented that bone marrow-derived endothelial and mesangial cells are integrated in the glomeruli, and contribute to microvascular repair. ${ }^{58}$ In this study, therefore, ARB may also directly affect bone marrow stem cells and/or progenitor cells, and may enhance repair processes in GN.
In this paper, we showed that ARB treatment inhibits the acute glomerular lesions, including capillary destruction, mesangial proliferation and activation, epithelial damage, and leukocyte infiltration in proliferative GN, and may prevent the progression of chronic kidney disease. ARB may diminish the injuries of individual glomerular cells in GN and may reduce macrophage infiltration through the angiotensin II receptors, with the alteration of these AT1R and AT2R expressions. 

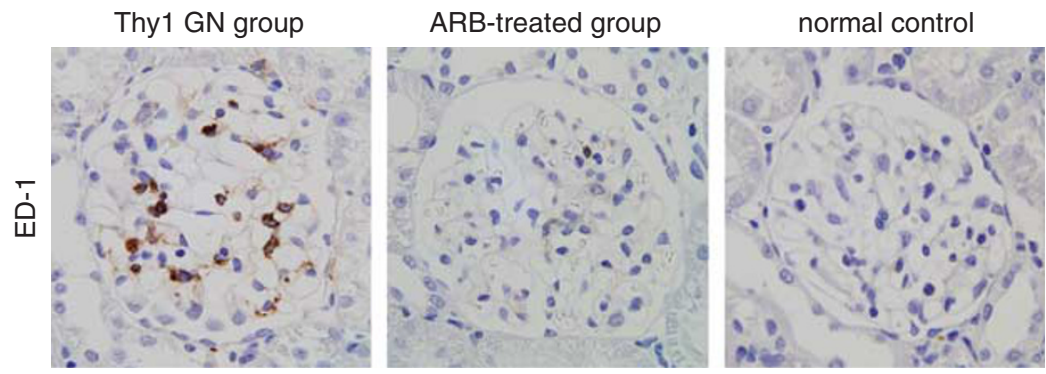

The number of ED-1(+) macropharges

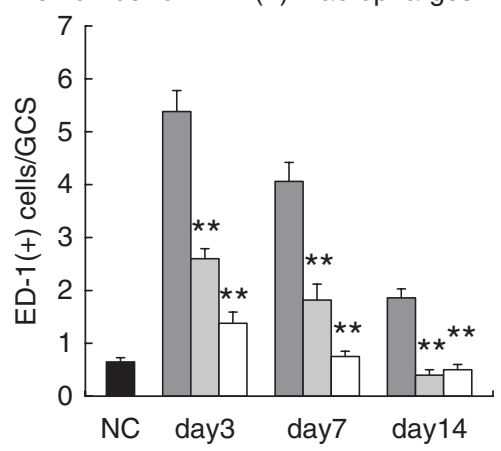

normal control group

Thy-1GN group

Thy-1GN+LD-ARB group

Thy-1GN+HD-ARB group

Figure 9 Glomerular infiltration of macrophages (ED-1) in Thy-1 GN, ARB-treated, normal control groups. ED-1: on day 3, ED-1-positive macrophages infiltrated into the glomeruli in the Thy-1 GN group. However, infiltration of ED-1-positive macrophages into glomeruli was decreased in ARB-treated groups. The number of ED-1-positive macrophages per glomerular cross section (GCS): ARB suppressed macrophage infiltration into glomeruli. Each column plot denotes the mean \pm s.e.. ${ }^{*} P<0.01$ vs Thy $1 G N$ group.

\section{ACKNOWLEDGEMENTS}

The authors wish to thank Adam Griesemer, MD, Transplantation Biology Research Center, Massachusetts General Hospital/Harvard Medical School, Boston, MA, for his critical review of the manuscript. The authors would like to thank Daiichi Sankyo Company (Tokyo, Japan) for generously providing Olmesartan. The authors also thank Mr Takashi Arai and Ms Mitsue Kataoka, Arimi Ishikawa, and Naomi Kuwahara for expect technical assistance.

1. Skeggs LT, Dorer FE, Kahn JR, et al. The biochemistry of the reninangiotensin system and its role in hypertension. Am J Med 1976;60:737-748.

2. Schmieder RE, Hilgers KF, Schlaich MP, et al. Renin-angiotensin system and cardiovascular risk. Lancet 2007;369:1208-1219.

3. Fogo $A B$. The role of angiotensin II and plasminogen activator inhibitor-1 in progressive glomerulosclerosis. Am J Kidney Dis 2000;35:179-188.

4. Brewster UC, Perazella MA. The renin-angiotensin-aldosterone system and the kidney: effects on kidney disease. Am J Med 2004;116:263-272.

5. Ruster C, Wolf G. Renin-angiotensin-aldosterone system and progression of renal disease. J Am Soc Nephrol 2006;17:2985-2991.

6. Wolf $G$. Renal injury due to renin-angiotensin-aldosterone system activation of the transforming growth factor-beta pathway. Kidney Int 2006;70:1914-1919.

7. Sachse A, Wolf $G$. Angiotensin II-induced reactive oxygen species and the kidney. J Am Soc Nephrol 2007;18:2439-2446.

8. Remuzzi A, Gagliardini E, Donadoni C, et al. Effect of angiotensin II antagonism on the regression of kidney disease in the rat. Kidney Int 2002;62:885-894.

9. Ma LJ, Nakamura S, Aldigier JC, et al. Regression of glomerulosclerosis with high-dose angiotensin inhibition is linked to decreased plasminogen activator inhibitor-1. J Am Soc Nephrol 2005;16: 966-976.

10. Griffin KA, Bidani AK. Progression of renal disease: renoprotective specificity of renin-angiotensin system blockade. Clin J Am Soc Nephrol 2006;1:1054-1065.
11. Shigematsu $\mathrm{H}$. Histological grading and staging of IgA nephropathy. Pathol Int 1997;47:194-202.

12. Lee HS, Lee MS, Lee SM, et al. Histological grading of IgA nephropathy predicting renal outcome: revisiting H.S. Lee's glomerular grading system. Nephrol Dial Transplant 2005;20:342-348.

13. Najafi CC, Korbet SM, Lewis EJ, et al. Significance of histologic patterns of glomerular injury upon long-term prognosis in severe lupus glomerulonephritis. Kidney Int 2001;59:2156-2163.

14. Szeto CC, Choi PC, To KF, et al. Grading of acute and chronic renal lesions in Henoch-Schonlein purpura. Mod Pathol 2001;14:635-640.

15. Weening JJ, D'Agati VD, Schwartz MM, et al. The classification of glomerulonephritis in systemic lupus erythematosus revisited. J Am Soc Nephrol 2004;15:241-250.

16. Bagchus WM, Hoedemaeker PJ, Rozing J, et al. Glomerulonephritis induced by monoclonal anti-Thy 1.1 antibodies. A sequential histological and ultrastructural study in the rat. Lab Invest 1986;55:680-687.

17. Ishizaki M, Masuda $Y$, Fukuda $Y$, et al. Experimental mesangioproliferative glomerulonephritis in rats induced by intravenous administration of anti-thymocyte serum. Acta Pathol Jpn 1986;36:1191-1203.

18. Shimizu A, Kitamura $H$, Masuda $Y$, et al. Apoptosis in the repair process of experimental proliferative glomerulonephritis. Kidney Int 1995;47:114-121.

19. Shimizu A, Masuda $Y$, Kitamura $H$, et al. Recovery of damaged glomerular capillary network with endothelial cell apoptosis in experimental proliferative glomerulonephritis. Nephron 1998;79: 206-214.

20. Shimizu A, Masuda $\mathrm{Y}$, Kitamura $\mathrm{H}$, et al. Complement-mediated killing of mesangial cells in experimental glomerulonephritis: cell death by a combination of apoptosis and necrosis. Nephron 2000;86: $152-160$.

21. Han GD, Suzuki K, Koike $H$, et al. IFN-inducible protein-10 plays a pivotal role in maintaining slit-diaphragm function by regulating podocyte cell-cycle balance. J Am Soc Nephrol 2006;17:442-453. 
22. Morioka $\mathrm{Y}$, Koike $\mathrm{H}$, Ikezumi $\mathrm{Y}$, et al. Podocyte injuries exacerbate mesangial proliferative glomerulonephritis. Kidney Int 2001;60: 2192-2204.

23. Hsu SM, Soban E. Color modification of diaminobenzidine (DAB) precipitation by metallic ions and its application for double immunohistochemistry. J Histochem Cytochem 1982;30:1079-1082.

24. Shimizu A, Masuda Y, Mori T, et al. Vascular endothelial growth factor165 resolves glomerular inflammation and accelerates glomerular capillary repair in rat anti-glomerular basement membrane glomerulonephritis. J Am Soc Nephrol 2004;15:2655-2665.

25. Nakajima $M$, Hutchinson $H G$, Fujinaga $M$, et al. The angiotensin II type 2 (AT2) receptor antagonizes the growth effects of the AT1 receptor: gain-of-function study using gene transfer. Proc Natl Acad Sci USA 1995;92:10663-10667.

26. AbdAlla S, Lother $\mathrm{H}$, Abdel-tawab AM, et al. The angiotensin II AT2 receptor is an AT1 receptor antagonist. J Biol Chem 2001;276: 39721-39726.

27. Suzuki K, Han GD, Miyauchi N, et al. Angiotensin II type 1 and type 2 receptors play opposite roles in regulating the barrier function of kidney glomerular capillary wall. Am J Pathol 2007;170:1841-1853.

28. Kobori H, Nangaku M, Navar LG, et al. The intrarenal renin-angiotensin system: from physiology to the pathobiology of hypertension and kidney disease. Pharmacol Rev 2007;59:251-287.

29. Bader M, Ganten D. Update on tissue renin-angiotensin systems. J Mol Med 2008;86:615-621.

30. Miyata N, Park F, Li XF, et al. Distribution of angiotensin AT1 and AT2 receptor subtypes in the rat kidney. Am J Physiol 1999;277:F437-F446.

31. Ozono R, Wang ZQ, Moore AF, et al. Expression of the subtype 2 angiotensin (AT2) receptor protein in rat kidney. Hypertension 1997;30:1238-1246.

32. Carey RM, Wang ZQ, Siragy HM. Role of the angiotensin type 2 receptor in the regulation of blood pressure and renal function. Hypertension 2000;35:155-163.

33. Wagner J, Gehlen F, Ciechanowicz A, et al. Angiotensin II receptor type 1 gene expression in human glomerulonephritis and diabetes mellitus. J Am Soc Nephrol 1999;10:545-551.

34. Wehbi GJ, Zimpelmann J, Carey RM, et al. Early streptozotocin-diabetes mellitus down regulates rat kidney AT2 receptors. Am J Physiol Renal Physiol 2001;280:F254-F265.

35. Bonnet F, Candido R, Carey RM, et al. Renal expression of angiotensin receptors in long-term diabetes and the effects of angiotensin type 1 receptor blockade. J Hypertens 2002;20:1615-1624.

36. Benigni A, Tomasoni S, Gagliardini E, et al. Blocking angiotensin II synthesis/activity preserves glomerular nephrin in rats with severe nephrosis. J Am Soc Nephrol 2001;12:941-948.

37. Amann K, Simonaviciene A, Medwedewa T, et al. Blood pressureindependent additive effects of pharmacologic blockade of the reninangiotensin and endothelin systems on progression in a low-renin model of renal damage. J Am Soc Nephrol 2001;12:2572-2584.

38. Manucha W, Oliveros L, Carrizo L, et al. Losartan modulation on NOS isoforms and COX-2 expression in early renal fibrogenesis in unilatera obstruction. Kidney Int 2004;65:2091-2107.

39. Nagai $\mathrm{Y}$, Yao L, Kobori $\mathrm{H}$, et al. Temporary angiotensin II blockade at the prediabetic stage attenuates the development of renal injury in type 2 diabetic rats. J Am Soc Nephrol 2005;16:703-711.

40. Remuzzi G, Benigni A, Remuzzi A. Mechanisms of progression and regression of renal lesions of chronic nephropathies and diabetes. J Clin Invest 2006;116:288-296.
41. Zoja C, Abbate M, Corna D, et al. Pharmacologic control of angiotensin II ameliorates renal disease while reducing renal TGF-beta in experimental mesangioproliferative glomerulonephritis. Am J Kidney Dis 1998;31:453-463.

42. Peters H, Border WA, Noble NA. Targeting TGF-beta overexpression in renal disease: maximizing the antifibrotic action of angiotensin II blockade. Kidney Int 1998;54:1570-1580.

43. Nakamura T, Obata J, Kimura $\mathrm{H}$, et al. Blocking angiotensin II ameliorates proteinuria and glomerular lesions in progressive mesangioproliferative glomerulonephritis. Kidney Int 1999;55:877-889.

44. Peters H, Border WA, Noble NA. Angiotensin II blockade and lowprotein diet produce additive therapeutic effects in experimental glomerulonephritis. Kidney Int 2000;57:1493-1501.

45. Nagamatsu T, Oka T, Nagao T, et al. Effects of KD3-671, an angiotensin II type 1 receptor antagonist, on anti-thy- 1 nephritis in rats. Biol Pharm Bull 2003;26:808-812.

46. Mahmood J, Khan F, Okada S, et al. Local delivery of angiotensin receptor blocker into the kidney ameliorates progression of experimental glomerulonephritis. Kidney Int 2006;70:1591-1598.

47. Liu N, Shimizu S, Ito-Ihara T, et al. Angiotensin II receptor blockade ameliorates mesangioproliferative glomerulonephritis in rats through suppression of CTGF and PAI-1, independently of the coagulation system. Nephron Exp Nephrol 2007;105:e65-e74.

48. Yu L, Border WA, Anderson I, et al. Combining TGF-beta inhibition and angiotensin II blockade results in enhanced antifibrotic effect. Kidney Int 2004;66:1774-1784.

49. Kondo S, Shimizu M, Urushihara M, et al. Addition of the antioxidant probucol to angiotensin II type I receptor antagonist arrests progressive mesangioproliferative glomerulonephritis in the rat. J Am Soc Nephrol 2006;17:783-794.

50. Okamura A, Rakugi $H$, Ohishi $M$, et al. Upregulation of reninangiotensin system during differentiation of monocytes to macrophages. J Hypertens 1999;17:537-545.

51. Ma J, Matsusaka T, Yang $\mathrm{H}$, et al. Local actions of endogenous angiotensin II in injured glomeruli. J Am Soc Nephrol 2004;15: 1268-1276.

52. Kakinuma $\mathrm{Y}$, Kawamura T, Bills $\mathrm{T}$, et al. Blood pressure-independent effect of angiotensin inhibition on vascular lesions of chronic renal failure. Kidney Int 1992;42:46-55.

53. Suganuma E, Zuo Y, Ayabe N, et al. Antiatherogenic effects of angiotensin receptor antagonism in mild renal dysfunction. J Am Soc Nephrol 2006;17:433-441.

54. Zambidis ET, Park TS, Yu W, et al. Expression of ACE (CD143) identifies and regulates primitive hemangioblasts derived from human pluripotent stem cells. Blood 2008;112:3601-3614.

55. Dezso K, Jelnes $\mathrm{P}$, Laszlo $\mathrm{V}$, et al. Thy- 1 is expressed in hepatic myofibroblasts and not oval cells in stem cell-mediated liver regeneration. Am J Pathol 2007;171:1529-1537.

56. Tuffin G, Huwyler J, Waelti E, et al. Drug targeting using OX7immunoliposomes: correlation between Thy1.1 antigen expression and tissue distribution in the rat. J Drug Target 2008;16:156-166.

57. Reinders ME, Rabelink TJ, Briscoe DM. Angiogenesis and endothelial cell repair in renal disease and allograft rejection. J Am Soc Nephrol 2006;17:932-942.

58. Rookmaaker MB, Smits AM, Tolboom H, et al. Bone-marrow-derived cells contribute to glomerular endothelial repair in experimental glomerulonephritis. Am J Pathol 2003;163:553-562. 